\title{
Conocimientos y buenas prácticas de manufactura en personas dedicadas a la elaboración y expendio de alimentos preparados, en el distrito de Los Olivos, Lima- Perú
}

Knowledge and good manufacturing practices in people engaged in the development and sale of prepared foods, in the district of Los Olivos, Lima - Peru

María Carrasco $^{1}$, Beder Guevara ${ }^{2}$, Néstor Falcón ${ }^{1}$,

\section{RESUMEN}

Objetivo: Evaluar el impacto de las capacitaciones sanitarias ofrecidas por un gobierno local sobre la persistencia y aplicaciones de los conocimientos de buenas prácticas de manufactura (BPM) en 60 manipuladores de alimentos de 11 mercados y 23 restaurantes del Distrito de los Olivos. Material y Métodos: Se utilizó un test post capacitación y dos meses después de la misma y se evaluó in situ la aplicación de las BPM en el manipulador de alimentos a través de una tabla de cotejo diseñada para el estudio. Resultados: Se encontró que los cursos de manipulación de alimentos aumentan el nivel de conocimientos sobre prácticas de manipulación de alimentos, pero que no necesariamente éstos se aplican en la preparación de los mismos. Se reportó que un aspecto crítico post capacitación fue el desconocimiento de la contaminación cruzada y sus implicancias. Se observó importantes omisiones en la aplicación de la BPM durante la manipulación de alimentos en la actividad rutinaria, entre ellas la inadecuada separación de alimentos en el momento de su preparación y la presencia de equipos inservibles en el lugar de trabajo. Conclusiones: Se hace necesario desarrollar capacitaciones constantes a fin de lograr la persistencia de los conocimientos sobre BPM y la supervisión de su aplicación por parte de los manipuladores de alimentos a fin de prevenir potenciales brotes de enfermedades transmitidas por alimentos.

PALABRAS CLAVE: ETA, BPM, manipuladores, capacitación, toxiinfección, alimentos

\section{SUMMARY}

Objective: The aim of the study was to assess the health impact of the sanitary training offered by a local government on the persistence and applications of knowledge of good manufacturing practices (GMP) in 60 food handlers from 11 markets and 23 restaurants in the District of Los Olivos. Material and methods: In order of that we used a post-training test and two months after training it was in situ assessed the application of GMP in the food handlers through a comparison table previously designed for the study. Results: The results show that food handling courses increase the level of knowledge about food handling practices, but that does not necessarily mean that they are applied in the food preparation, reporting that a critical post-training was the lack of cross-contamination and its implications. It was found significant omissions in the application of the GMP during food handling in the routine

Facultad de Medicina Veterinaria y Zootecnia, Universidad Peruana Cayetano Heredia. Lima, Perú.

Municipalidad Distrital de Los Olivos. Lima - Perú. 
activity, including inadequate separation of food at the time of its preparation and the presence of non-functional equipment in the workplace. Conclusions: It is necessary to develop continuous training to achieve the persistence of GMP knowledge and supervision of its application by the food handlers in order to prevent food-borne diseases

KEYWORDS: food-borne diseases, GMP, food handler, training, food poisoning, food.

\section{INTRODUCCIÓN}

La venta de alimentos en la vía pública es una práctica tradicional en América Latina y el Caribe, representando una fuente de ingresos regulares para millones de personas. Esta práctica puede resultar un riesgo para la salud de los consumidores cuando es realizada por personas con limitada educación y competencias en la transformación de alimentos (1). Las malas prácticas de manipulación puede conllevar a la contaminación bacteriana de los alimentos, factor que representa uno de los riesgos más importante asociado a la aparición de brotes infecciosos en América Latina y el Caribe (2). Estas infecciones reciben el nombre de enfermedades transmitidas por los alimentos (ETA) y ocurren como consecuencia de la falta de higiene en la manipulación y procesamiento de los alimentos o después de la preparación de los mismos (3-4).

En el Perú, las ETA afectan principalmente a los sectores más deprimidos de la población. Más del $90 \%$ de las mismas se originan por el consumo de alimentos en escuelas, mercados, venta pública e incluso en el propio hogar (5). Un estudio en 27 restaurantes (especializados en la preparación de alimentos a base de pescado), ubicados en 21 distritos de Lima Metropolitana, encontró que en el $40 \%$ de los establecimientos el personal de cocina tenía la costumbre de comer o beber mientras trabajaba, el $76 \%$ del personal de cocina y salón no estaban correctamente uniformados, el $64 \%$ de los trabajadores estaban desaseados y el $77 \%$ de los locales no tenían letreros educativos para la higiene de los comensales, entre otras prácticas antihigiénicas (6). En otro estudio realizado en el distrito de Comas se observó que el 98\% de los puestos de venta de alimentos no tenían depósitos para la eliminación de los residuos sólidos y que el $72 \%$ de los manipuladores carecían de capacitación en prácticas higiénicas de manipulación de alimentos (7).

Las medidas preventivas a la inadecuada manipulación de los alimentos incluyen la transferencia de conocimientos y prácticas para mejorar las buenas prácticas de manufactura (BPM) en personas dedicadas a la elaboración y expendio de alimentos (8). Ello se encuentra estipulado en el Reglamento sobre Vigilancia y Control Sanitario de Alimentos y Bebidas en el Perú, específicamente en los artículos 49 y 50 (aseo y presentación personal), 52 (higiene personal), y 53 y 55 (recibir capacitación en higiene de alimentos basadas en BPM) (9).

La eficacia de los cursos de capacitación para manipuladores de alimentos ha sido evaluada en diferentes estudios. En España, se realizó una evaluación pre y post capacitación, encontrándose que la mayoría de los manipuladores conocían qué hábitos eran considerados poco higiénicos; sin embargo, ello no garantizaba que estos no se realizaran en la práctica (10). En Colombia se encontró que el 50,3\% de los manipuladores de alimentos ingresó a trabajar con sólo el examen médico y $60,7 \%$ realizó el curso de manipulación de alimentos. En la práctica se evidenció que el $17 \%$ manejó simultáneamente dinero y alimentos, $15.2 \%$ uso joyas, $8.9 \%$ tenían uñas largas y con esmalte y el $15.2 \%$ refirieron no lavarse las manos tras la manipulación de dinero (11). En Venezuela, se encontró que $84,2 \%$ de los empleados declararon haber asistido a talleres sobre manipulación de alimentos, pero el $68,9 \%$ de ellos no pudieron demostrar tener un nivel de conocimiento satisfactorio (12).

Entendiendo que el manipulador de alimentos juega un rol central en la calidad e innocuidad de los alimentos que se expenden, las Municipalidades han implementado cursos de formación con la finalidad de desarrollar hábitos, actitudes y practicas higiénicas en ellos.

En este contexto, el objetivo del presente estudio fue evaluar el impacto de las capacitaciones sanitarias ofrecidas por un gobierno local sobre la persistencia $\mathrm{y}$ aplicaciones de los conocimientos de buenas prácticas de manufactura (BPM) en 60 manipuladores de alimentos de 11 mercados y 23 restaurantes del Distrito de los Olivos

\section{MATERIAL Y MÉTODOS}

El presente estudio se desarrolló en establecimientos 
de venta de alimentos (23 restaurantes y 11 puestos de mercados) del distrito de Los Olivos en Lima, Perú. El análisis de datos se realizó en la Facultad de Medicina Veterinaria y Zootecnia de la Universidad Peruana Cayetano Heredia, correspondiendo el mismo a un modelo de investigación observacional descriptiva, con mediciones en series de Tiempo.

La población objetivo fueron los manipuladores de alimentos de restaurantes y mercados del distrito Los Olivos y la muestra estuvo conformada por 60 personas (30 trabajadores de restaurantes y 30 de puestos de comida en mercados).

Para recolectar la información se utilizó un cuestionario de evaluación de conocimientos de diez preguntas, el mismo que utiliza el área de Salubridad y Saneamiento Ambiental de la Municipalidad de Los Olivos para sus actividades de capacitación. El cuestionario fue aplicado en dos momentos: inmediatamente después y a los dos meses posteriores a la capacitación. Las BPM fueron evaluadas mediante una tabla de cotejo, la que fue elaborada y validada especialmente para la investigación. Esta se elaboró a partir de las informaciones que se brindaron durante la capacitación a los manipuladores.

Los datos fueron analizados mediante estadísticas descriptivas. Las variables cualitativas (características que describen a la población en estudio) y los resultado de la tabla de cotejo se resumieron en tablas de frecuencia. Para la determinación de diferencias en los puntajes entre las evaluaciones se utilizó la prueba de $\mathrm{T}$ de Student para muestras apareadas. Para el análisis de datos se utilizó el programa estadístico SPSS 15.0.

\section{RESULTADOS}

Se evaluó a 60 manipuladores de alimentos de los cuales $17(28,3 \%)$ fueron varones y $43(71,7 \%)$ mujeres. La edad promedio de los manipuladores fue de 40,3 años, con una desviación estándar de 12,2 y un rango de 18 a 66 .

Las preguntas que tuvieron un porcentaje de respuestas correctas igual o menor al $80 \%$ en la evaluación realizada inmediatamente después de la capacitación fueron: ¿Cómo se genera una contaminación cruzada?, ¿Qué es un manipulador de alimento? y ¿Qué son las ETA?, las mismas que superaron ese límite en la evaluación realizada dos meses después de la capacitación. La única pregunta que tuvo un porcentaje de respuestas correctas inferior al $80 \%$ en la evaluación realizada dos meses posteriores a la capacitación fue la que solicitaba recomendaciones para el cuidado de los alimentos. Sin embargo, las notas obtenidas por los manipuladores de alimento entre el primer y segundo examen fueron estadísticamente similares. (Tabla 1).

La evaluación de las BPM reportó que las prácticas positivas que se cumplieron en un porcentaje menor

Tabla 1. Frecuencia de respuestas correctas en la prueba de conocimiento sobre buenas prácticas de manejo aplicado a manipuladores de alimentos en mercados y restaurantes del distrito de Los Olivos, Lima, Perú, 2011 (n=60).

\begin{tabular}{|c|c|c|c|c|c|}
\hline & \multirow[t]{2}{*}{ Preguntas* } & \multicolumn{2}{|c|}{$\begin{array}{l}\text { Al final de la } \\
\text { capacitación }\end{array}$} & \multicolumn{2}{|c|}{$\begin{array}{c}\text { Dos meses post la } \\
\text { capacitación }\end{array}$} \\
\hline & & $\mathrm{N}^{\mathrm{o}}$ & $\%$ & $\mathrm{~N}^{\circ}$ & $\%$ \\
\hline 1. & ¿Dónde deben colocarse los residuos sólidos (basura)? & 59 & 98,3 & 53 & 88,3 \\
\hline 2. & ¿De qué material debe de ser la tabla de picar? & 60 & 100,0 & 56 & 93,3 \\
\hline 3. & ¿Qué es un manipulador de alimento? & 45 & 75,0 & 53 & 88,3 \\
\hline 4. & ¿Cuándo debe lavarse las manos el manipulador de alimentos? & 50 & 83,3 & 52 & 86,7 \\
\hline 5. & ¿Qué indumentaria debe de usar el manipulador de alimentos? & 57 & 95,0 & 56 & 93,3 \\
\hline 6. & ¿Quiénes deben de obtener el carné de sanidad? & 50 & 83,3 & 58 & 96,7 \\
\hline 7. & ¿Con que frecuencia se renueva el carné de sanidad? & 59 & 98,3 & 57 & 95,0 \\
\hline 8. & ¿Qué son las ETA? & 48 & 80,0 & 52 & 86,7 \\
\hline 9. & ¿Cómo se genera una contaminación cruzada? & 40 & 66,7 & 52 & 86,7 \\
\hline & ¿Recomendaciones para el cuidado de los alimentos? & 55 & 91,7 & 46 & 76,7 \\
\hline
\end{tabular}

*La forma como se presentaron las preguntas a los encuestados difiere de la que se presenta en el cuadro, sin que ello haya variado el sentido de las mismas. 
Tabla 2. Resultados de la evaluación de prácticas de manejo en manipuladores de alimentos en mercados y restaurantes del distrito de Los Olivos, Lima, Perú, 2011 ( $n=60)$.

\begin{tabular}{|c|c|c|c|}
\hline & Prácticas Evaluadas & $\mathrm{N}^{\mathrm{o}}$ & $\%$ \\
\hline \multicolumn{4}{|c|}{ Prácticas positivas: } \\
\hline 1. & Porta su carnet sanitario. & 55 & 91,7 \\
\hline 2. & Porta certificado de capacitación de manipulador de alimento. & 60 & 100,0 \\
\hline 3. & Utiliza gorro blanco y limpio. & 29 & 48,3 \\
\hline 4. & Tiene el cabello corto y/o recogido. & 57 & 95,0 \\
\hline 5. & $\begin{array}{l}\text { Mujeres: no usa maquillaje o usa maquillaje discreto // Hombres: están bien } \\
\text { afeitados. }\end{array}$ & 59 & 98,4 \\
\hline 6. & Tiene las uñas cortas, sin esmalte, limpias y sin joyas (anillos, pulseras, relojes). & 45 & 75,0 \\
\hline 7. & No presenta problemas de onicomicosis. & 59 & 98,4 \\
\hline 8. & Usa chaqueta y/o mandil blanco y limpio. & 41 & 68,4 \\
\hline 9. & Usa ropa interna limpia. & 41 & 68,4 \\
\hline 10. & Usa zapatos cerrados y limpios. & 49 & 81,7 \\
\hline & $\begin{array}{l}\text { Utiliza o coloca los residuos sólidos en un tacho adecuado, con una bolsa inte- } \\
\text { rior de plástico y con tapa. }\end{array}$ & 35 & 58,3 \\
\hline 12. & Mantiene limpia la tabla de picar que utiliza. & 51 & 85,0 \\
\hline & $\begin{array}{l}\text { Mantiene la adecuada separación de los alimentos en el momento de su prepara- } \\
\text { ción, para evitar contaminación cruzada de los alimentos. }\end{array}$ & 46 & 76,7 \\
\hline 14. & Mantiene los productos de limpieza y desinfectantes separados de los alimentos. & 52 & 86,7 \\
\hline
\end{tabular}

\section{Prácticas negativas}

1. Permite la presencia de animales en la cocina.

2. Permite la presencia de equipos inservibles o en desuso como cartones, costales, etc.

3. Se observa la presencia del manipulador con algún síntoma de enfermedad.

4. Se observa algún tipo de acción - mal habito (escupir, comer, toser, rascarse la cabeza, arreglarse el cabello, tocar o secarse el sudor del rostro, fumar, etc.) durante la preparación de alimentos.

610,0

al $80 \%$ fueron: el uso de gorro blanco y limpio, el colocar los residuos sólidos en un tacho adecuado revestido con bolsa interior de plástico y con tapa, el uso de chaqueta y/o mandil blanco y limpio, el uso de ropa interna limpia, el tener las uñas cortas, limpias y sin esmalte, el no usar joyas (anillos, pulseras, relojes), y el mantener una adecuada separación de los alimentos en el momento de su preparación para evitar contaminación cruzada entre alimentos de diferente tipo y origen. Entre las prácticas negativas, la más común fue observar la presencia de equipos inservibles o en desuso como cartones, costales, etc. Dentro de las áreas de trabajo y procesamiento (Tabla 2).

\section{DISCUSIÓN}

La necesidad de proveer a los consumidores alimentos seguros y nutritivos para satisfacer sus necesidades en forma rápida hace que cada vez más un mayor número de ellos recurra a mercados, restaurantes o ferias agropecuarias. El buen funcionamiento de estos lugares es por ello regulado por instituciones públicas como el Ministerio de Salud en su rol normativo y las Municipalidades en su función operativa (3).

Los resultados del estudio muestran que a esta actividad se dedican personas de ambos sexos y de diferentes edades, quienes tienen la responsabilidad de proveer alimentos sanos a los consumidores. 
Para ello recibieron una capacitación que les debió haber permitido desarrollar prácticas de manejo y almacenamiento de insumos, y de manipulación adecuada de los mismos para la preparación de los alimentos. Se espera que con ello el cumplimiento de las BPM disminuya los riesgos de ocurrencia de ETA en la población consumidora de alimentos preparados (13).

En general, el hallazgo de respuestas correctas en la evaluación de conocimiento sobre BPM realizado inmediatamente después de finalizada la capacitación y a los dos meses después de esta permitió a los manipuladores participantes tener conocimientos acerca del tema y que estos inclusive permaneciesen en el tiempo. Sin embargo, existen algunos conocimientos a los que se requiere poner mayor atención con el fin de reforzarlos al momento de las capacitaciones.

Las preguntas que tuvieron un porcentaje de respuesta correcta similar o por debajo del $80 \%$ en la evaluación realizada inmediatamente después de la capacitación se pueden considerar como críticas. El hecho de conocer cómo se genera una contaminación cruzada es muy importante ya que su desconocimiento podría acarrear una contaminación de los alimentos por microorganismos patógenos a través de alimentos, utensilios y superficies en general y en consecuencia favorecer la presentación de ETA. Así mismo, el conocer la importancia de su rol como manipuladores de alimentos y el saber que son las ETA, son dos conceptos sumamente críticos. En el primer caso, existió un grupo de trabajadores que no se reconocieron como manipuladores de alimentos (25\%) y otro $(20 \%)$ que desconocía el significado del vocablo ETA. La conjunción de ambas es sumamente grave por las implicancias que esto tiene en el reconocer y asumir su responsabilidad en evitar la transmisión de enfermedades por la vía digestiva.

Es importante destacar que el porcentaje de aciertos encontrados en la evaluación realizada dos meses después de la capacitación, en estos casos, aumenta, lo que puede haberse debido a que los manipuladores de alimentos recibieron material educativo que pudieron haber seguido revisando más allá de la etapa de capacitación. Esta es una práctica muy favorable.

En contraste a lo anterior, al solicitarle a los manipuladores que mencionen las recomendaciones para el cuidado de los alimentos, se ve que el recordarlas disminuye entre la primera y segunda evaluación, lo cual puede representar un potencial riesgo para la salud de los consumidores debido a que su desconocimiento, en el mediano o largo plazo, podría estar asociado también a su omisión durante el trabajo.

Aunque las respuesta afirmativas a la mayoría de preguntas estuvieron por encima del $80 \%$, es alarmante que alrededor del $15 \%$ de los manipuladores desconociese o no respondiese correctamente a la pregunta referente a la importancia de lavarse las manos antes, durante y después del trabajo, tanto inmediatamente después de la capacitación como dos meses más tarde. Ello, aun en su insignificancia numérica, es un valor grave si se tiene en cuenta que la falta de higiene conlleva al hallazgo frecuente de cepas enterotoxigénicas en las manos de los manipuladores de alimentos. Es importante por ello resaltar en futuras capacitaciones el énfasis que debe ponerse en la higiene personal, principalmente en el correcto lavado de las manos, sin descuidar la importancia de las BPM, para prevenir brotes de ETA provocadas por este tipo de microorganismos (14).

Resultados similares sobre el impacto de las capacitaciones a manipuladores de alimento se han encontrado en otros estudios en donde la eficacia de los cursos de formación sanitaria fue igualmente baja $(10,12)$.

Al evaluar la implementación de las BPM in situ se encontró que menos del $80 \%$ de los manipuladores de alimentos usaba correctamente su uniforme. En este grupo de prácticas se encuentra el uso de gorro y chaqueta blanco y limpio, así como el uso de ropa interna limpia, lo cual contrasta significativamente con los resultados obtenidos en la evaluación de conocimientos en donde la mayoría de encuestados respondió correctamente las preguntas correspondientes a este rubro.

Otra práctica asociada a la higiene personal se refiere al hecho de tener las uñas cortas, limpias y sin esmalte, además del no uso de joyas (anillos, pulseras, relojes). Se ha demostrado que las uñas artificiales (acrílicas) pueden ser medios potenciales de transmisión de patógenos a los alimentos (15). En el caso de enfermeros y otros profesionales de salud se ha encontrado que el uso de las uñas cortas reducía significativamente el riesgo de colonización de bacterias como Pseudomonas aeruginosa (16). En la población de este estudio se encontró en muy baja presencia onicomicosis, lo que a su vez redujo la incidencia de este problema como factor condicionante 
de un adecuado desempeño de sus funciones (17).

El manejo adecuado de la basura sólida fue igualmente deficiente. Poco más del 50\% cumple correctamente con esta práctica, por lo que en la mayoría de los casos es una deficiencia que puede favorecer la contaminación de los alimentos y como consecuencia aumentar el riesgo de diseminación de las ETA. Sin embargo, este escenario es menos crítico a otro estudio realizado anteriormente en Comas, Lima - Perú, donde se encontró que el 98.4\% de los puestos de venta de alimentos en la vía pública no contaban con un depósito adecuado para la eliminación de los residuos sólidos (7).

También la adecuada separación de los alimentos en el momento de la preparación era deficiente. Se debe tener en cuenta que la contaminación muchas veces no ocurre de forma directa, sino a través del contacto con otros alimentos crudos, o a través de utensilios, insectos o superficies sucias (18). Se ha demostrado que los vegetales crudos son portadores o vehículos para patógenos como bacterias, virus y parásitos, y que su eliminación por métodos convencionales de lavado no es totalmente efectivo (19), por lo que la separación de los alimentos durante su preparación es sumamente importante.

Asociado al riesgo de contaminación cruzada está también el uso de las tablas de picar. Si bien es cierto que en la mayoría de los casos estos implementos de cocina estaban limpios, también es cierto que el material con el cual estos son fabricados (madera) no era el más recomendable debido a su porosidad, en la cual se pueden albergar microorganismos y de donde no pueden ser fácilmente eliminados por los procedimientos convencionales de desinfección. Los microorganismos sobreviven en tablas de madera en estado latente por largo tiempo y en el próximo uso, estos pueden contaminar otros alimentos (20). La Norma Sanitaria para el funcionamiento de restaurantes y servicios afines, recomienda que las tablas de picar sean de material inabsorbente y de superficie lisa y que se mantengan en buen estado de conservación e higiene.

Los productos de limpieza y desinfección también pueden ocasionar contaminación cruzada. Estos contienen agentes químicos peligrosos que si entran en contacto con los alimentos o se encuentran en zonas donde se manipulan alimentos, pueden afectar la salud de los consumidores. Malos hábitos como el manejar dinero, desinfectantes, ollas, etc., así como el contacto de los alimentos con aerosoles de los trabajadores (estornudos), vómitos, lesiones en las manos, también pueden conllevar a una contaminación cruzada (21).

Se observó la presencia de animales dentro de las cocinas de los restaurantes, así como de equipos inservibles como cartones, costales, etc. Estos, además de atraer plagas, representan focos de contaminación y propagación de microorganismos para los alimentos. Finalmente, entre las prácticas negativas, aunque en menor porcentaje de ocurrencia, se dieron casos en que los operarios no contaban con sus respectivos carnets sanitario al momento de la inspección o lo tenían vencido, lo cual no hizo sino demostrar que por un lado no existe responsabilidad de parte del manipulador en cumplir esta exigencia, ni tampoco por parte de los funcionarios de las municipalidades en hacerla cumplir.

En términos generales se puede afirmar que los buenos resultados de las evaluaciones de conocimiento no guardan relación con lo que en la práctica se puede observar. Esto debe obligar a insistirse en la importancia de aplicar los conocimientos obtenidos en las capacitaciones teóricas, tanto durante las capacitaciones en sí como después de las mismas a fin de garantizar su cabal cumplimiento. Esto favorecerá la probabilidad de disminuir las fuentes de contaminación de los alimentos y como consecuencia los casos de ETA, garantizando a la población en general un suministro de alimentos nutritivos, limpios, de calidad e inocuos.

\section{CONCLUSIONES}

A partir de los resultados del estudio se concluye que los cursos de capacitación para manipuladores de alimentos permiten alcanzar y mantener un buen nivel de conocimientos sobre las buenas prácticas de manipulación; sin embargo, éste es principalmente teórico y no necesariamente se traduce en una mejor labor diaria por parte de los beneficiarios. Por ello, se recomienda que las capacitaciones futuras incluyan tópicos tanto teóricos como prácticos, o mejor aún, que sean eminentemente prácticos (entrenamientos en servicio bajo el concepto de aprender haciendo) complementados con los fundamentos teóricos necesarios, y que además se cuente con los sistemas de supervisión y seguimiento que realmente garanticen su aplicación permanente. 


\section{Correspondencia}

María Carrasco Sánchez

Correo electrónico: ariam_1@hotmail.com

\section{REFERENCIAS BIBLIOGRAFICAS}

1. Organización de las Naciones Unidas para la Agricultura y la Alimentación. Buenas Prácticas de Higiene en la preparación y venta de los alimentos en la vía pública en América Latina y el Caribe. Roma, Italia: FAO. 2009.p.180.

2. Organización Panamericana de la Salud. La Salud en las Américas. Publicación Científica y Técnica No. 587. Washington, D.C.: Organización Panamericana de la Salud. 2009. p. 473.

3. Organización de las Naciones Unidas para la Agricultura y la Alimentación, Organización Mundial de la Salud. Programa de Mercados y Restaurantes Saludables de la red de municipios y comunidades saludables de Lima y Callao (Internet). San José, Costa Rica: FAO/OMS; 2005. (Citado en Mayo del 2011) Disponible en: http://www.bvsde.paho.org/ bvsacd/cd65/af265s.pdf

4. Organización de las Naciones Unidas para la Agricultura y la Alimentación. Enfermedades socioeconómicas transmitidas por alimentos y su impacto: Estudios de caso en Costa Rica, El Salvador, Guatemala, Honduras y Nicaragua. FAO. 2009. p. 187.

5. Ministerio de Salud. Lineamiento del Ministerio De Salud: Estrategia institucional y propuestas sectoriales para el periodo 2001- 2006. Lima, Perú: MINSA; 2002.

6. Centro de Promoción de la Pequeña y de la Microempresa. Manual de Buenas Prácticas de Manipulación: dirigido a empresarios, administradores y empleados de restaurantes. Lima, Perú: PromPyme; 2001.p. 82.

7. Quispe J, Sánchez V. Evaluación Microbiológica y Sanitaria de puestos de venta ambulatoria de alimentos del distrito de Comas, Lima-Perú. Rev Perú Med Exp. salud pública. 2001; 18: 27-32.

8. Maestre M, Muñoz S. Medidas de actuación para la prevención de la toxiinfección alimentaria. Rev Med Segur Trab. 2008; 54:121-130.

9. Presidencia de la República. Decreto Supremo $N^{\circ}$ 007-98-SA. Reglamento sobre Vigilancia y Control Sanitario de Alimentos y Bebidas. Lima, Perú: Diario oficial El Peruano; 25 de setiembre de 1998.

10. Gil de Vergara PV, Colomer C, y Serra Ll. Evaluación de la eficacia de los cursos de formación sanitaria dirigidos a los manipuladores de alimentos de un área sanitaria. Rev Esp Sal Pub 2000; 74:299-307.
11. Flórez A.C, Rincón C, Garzón P, Vargas N, Enríquez C. Factores relacionados con enfermedades transmitidas por alimentos en restaurantes de cinco ciudades de Colombia. Asociación Colombiana de Infectología. 2007; 12(4): 255-266.

12. Iriarte M, Fermi O. Evaluación del conocimiento sobre buenas prácticas de manipulación de alimentos del personal de cocina de los hoteles 5 estrellas, Isla de Margarita, Venezuela. Rev. INHRR. 2001; 34:17-22.

13. Serna L, Correa M. Evaluación de la inocuidad de los alimentos consumidos por una población de niños y adultos mayores. Rev Alimentos Hoy. 2011; 1-12.

14. Gubbay L, Galanternik L, Galan G, Cabrera J, Durango, M. Staphylococcus aureus: Sensibilidad antibiótica y detección de enterotoxinas de cepas aisladas de alimentos y manos de manipuladores. Rev de Cien. 2004; 30:12-4.

15. Wachukwu C, Abbey S, Ollor A, Obilor N. Public health implication of artificial finger nails used by health workers and food handlers in Port Harcourt, Nigeria. Journal of Applied Sciences. 2007; 7: 35803583.

16. Hedderwick S, Mcneil S, Lyons M, Kauffman C. Pathogenic organisms associated with artificial fingernails worn by health care workers. Infect Control Hosp Epidemiol. 2000; 21: 505-509.

Ballesté R, Mousqués N, Gezuele E. Onicomicosis. Revisión del tema. Rev Med Uruguay. 2003; 19: 93106.

17. Organización Panamericana de la Salud. Las 5 claves para mantener los alimentos seguros. INCAP/OPS. 2006. p. 34.

18. Merino LA. Importancia de los alimentos que se consumen crudos en la transmisión de enfermedades de origen alimentario (Internet). SIIC; 2005. (Citado el 21 de enero de 2012). Disponible en: http://www. siicsalud.com/dato/dat043/05504016.htm

19. Misner S. Cutting boards (Plastic vs. Wood). Safe food handling: Be smart (Internet). Arizona: College of Agriculture \& Life Sciences, The University of Arizona; 2008. (Citado el 21 de enero del 2012) Disponible en : http://cals.arizona.edu/pubs/health/ az1076.pdf

20. Todd E, Greig J, Bartleson C, Michaels BS. Outbreaks where food workers have been implicated in the spread of foodborne disease. Part 6. Transmission and survival of pathogens in the food processing and preparation environment. J Food Prot. 2009; 72(1):202-19.

\begin{tabular}{c|}
\hline Recibido: $31 / 01 / 2013$ \\
Aceptado: $24 / 06 / 2013$ \\
\hline
\end{tabular}

